\title{
Efficient Pourbaix diagrams of many-element
}

\section{compounds}

\author{
Anjli Patel, ${ }^{\dagger}$ Jens K. Nørskov, ${ }^{\dagger, \ddagger}$ Kristin Persson, ${ }^{\llbracket}$ and Joseph H. Montoya*,\| \\ $\dagger$ Department of Chemical Engineering, Stanford University, Stanford, CA \\ $\ddagger$ Department of Physics, Danish Technical University, Lyngby, Denmark \\ \Lawrence Berkeley National Laboratory, Berkeley, CA \\ $\S$ Department of Materials Science, University of California, Berkeley, CA \\ \| Toyota Research Institute, Los Altos, CA \\ E-mail: joseph.montoya@tri.global
}

\begin{abstract}
Pourbaix diagrams are an invaluable tool for exploring the corrosion profiles of materials as a function of ambient $\mathrm{pH}$ and electrochemical potential. ${ }^{1}$ In recent years, high-throughput computational materials science efforts like those from the Materials Project ${ }^{[23}$ have enabled more comprehensive Pourbaix diagrams to be constructed and disseminated from computational data. $\cdot \frac{455}{15}$ These analyses have informed a number of computational studies of materials for electrochemical applications, aqueous electrocatalysis ${ }^{6-9}$ and photoelectrocatalysis, 10 ? 11 non-equilibrium crystallization,, 1213 and corrosion-resistant alloy design. ${ }^{14115}$ In these, pourbaix analysis of multi-element systems is particularly valuable, as finding elusive materials like acid-stable oxygen evolution catalysts, ${ }^{17}$ earth-abundant hydrogen evolution catalysts,,${ }^{16}$ and selective $\mathrm{CO}_{2}$ reduction catalysts ${ }^{[17}$ has and will likely continue to require exploration and optimization in multi-element spaces. However, Pourbaix analysis of phase stability on these resources have been limited to 3 or fewer elements, largely because computing the electrochemical phase stability of higher composition spaces has proven inefficient with existing
\end{abstract}


methods.

In this report, we provide details of a modified method for Pourbaix diagram construction which enables diagrams to be constructed efficiently in much higher compositional spaces, which enables phase stability analysis of similarly complex individual materials. We demonstrate this functionality with an analysis of the phase stability of a complex material for alkaline oxygen evolution (OER) and highlight our implementation in the open-source pymatgen ${ }^{18}$ code and on the Materials Project website (materialsproject.org).

The primary bottleneck in pymatgen's prior implementation of multi-element Pourbaix diagrams resides in their pre-processing iteration over potential combinations of compounds. Essentially, the current method, based on the thermodynamic formalisms outlined in ref $\frac{19}{19}$ and ref ${ }^{4}$ is to iterate over all valid stoichiometric combinations of compounds in the chemical system which satisfy the compositional constraint particular to a given Pourbaix diagram (e.g. $\mathrm{Fe}: \mathrm{Cr}=2: 1)$.

In this scheme the scaling of Pourbaix diagram construction occurs with $\mathrm{n}$ choose $\mathrm{m}$, where $\mathrm{n}$ is the number of compounds included and $\mathrm{m}$ is the number of elements included. Since larger numbers of elements tend to produce more entries on queries of the database, Pourbaix diagrams become prohibitively expensive after 3 elements. More explicitly, 4 or 5 element Pourbaix diagrams for the Ba-Sr-Co-Fe (present in BCSF, $\stackrel{20}{20}$ an alkaline OER catalyst), Al-Cu-Mn-Mg-Fe (present in some commercial Duralumin alloys) would require $\sim 10^{9}$ and $\sim 10^{11}$ evaluations of selected combinations of compounds from the pool of materials.

Considerable speedup is achieved by filtering for entries on the convex hull of the solid compositional phase diagram, which is at least partially motivated by physical reasoning that those materials should appear in the Pourbaix diagram absent any ions. This process, however, is complicated by the variable chemical potential of $\mathrm{H}^{+}$and $\mathrm{e}^{-}$on the Pourbaix diagram (but not on the compositional phase diagram) and the need to add ionic species, which still results in poor combinatoric scaling. The process was also further improved (e.g. in pymatgen) by virtue of it being easily parallelized, but this still only renders a factor of $\mathrm{N}$ 
speedup when much larger factors are required for the higher-element spaces to be tractable. In summary, with currently hardware, execution times for 5-element and higher diagrams are estimated to be on the order of years.

To pre-filter the Pourbaix compounds that may appear on the hull, one can compute the convex hull in a similar manner as a pymatgen-implemented grand-canonical phase diagram, but in a space which includes fractional coefficients on electrons and protons. This essentially amounts to a grand-canonical phase diagram in $\mathrm{H}^{+}-\mathrm{e}^{-}-\mathrm{H}_{2} \mathrm{O}-\mathrm{M}_{1}-\mathrm{M}_{2}-\ldots-\mathrm{M}_{n}$, for which valid stable (i. e. minimal free energy of formation) compounds can be found by taking the convex hull in the space where $\mu_{\mathrm{H}^{+}}$and $\mu_{\mathrm{e}^{-}}$are treated as free variables (i.e. points corresponding to their reference energies are not include in the convex hull point inputs). For the purposes of finding stable combinations of entries, a 4-D convex hull and its corresponding simplicies in $N_{p H}-N_{\Phi}-x_{1}-x_{2} \ldots x_{n-1}$, where $N_{p H}$ and $N_{\Phi}$ are scaling factors for the Pourbaix energy ${ }^{12}$ with respect to $\mathrm{pH}$ and applied potential, and $x_{n}$ are non-OH fractions of the non-OH elemental composition, are sufficient. This hull and its corresponding simplices are illustrated for the La-Co Pourbaix system in Figure 2. Under the assumption of ideal mixing, decomposition products in this space correspond to simplices on the convex hull, meaning that valid Pourbaix decomposition products can be limited to those which appear in a given simplex. The precise reduction in scaling will depend on the complexity of this hull, but it allows the combinatorial complexity to be isolated only to existing facets. In practice, this offers a reduction in the number of iterations by 2-3 orders of magnitude (see benchmarking in Figure 11).

We also note here that the determination of the Pourbaix regions in which the free energies of the corresponding species is minimal are determined from a halfspace intersection of 2-dimensional planes corresponding to the pre-processed "multi-entry" phases (as termed in pymatgen), which differs from the grid-based methods implemented in $\mathrm{ASE}^{21}$ and from ref. ${ }^{14}$ However, our preprocessing might also be used to pre-filter compounds in a grid-based approach as these to reduce the iterative load at each evaluated point in E-pH space. 
To illustrate the power of the method, we benchmark the timing on the Pourbaix diagram derived from the compound with the highest number of elements in the Materials Project database $\left(\mathrm{Ba}_{2} \mathrm{NaTi}_{2} \mathrm{MnRe}_{2} \mathrm{Si}_{8} \mathrm{HO}_{26} \mathrm{~F}\right.$, mp-1215061), which completes in 15-20 minutes. This points to the added capability of featurizing the entire MP dataset with Pourbaix decomposition grids, which might make Pourbaix diagrams more amenable to emerging data-intensive prediction methods such as machine and deep learning.

To illustrate with another practical example, we include the heatmap corresponding to the decomposition energy against the Pourbaix diagram (using the methods developed in in ref ${ }^{5}$ ) of the Ba-Sr-Co-Fe chemical system, a model system for the BSCF catalyst known for its high activity as an alkaline OER catalyst. $\frac{20}{20}$ The Pourbaix diagram reveals that cation leaching is thermodynamically favorable with a modest driving force, suggesting that the material may not dissolve completely, but that the near-surface region may partially decompose in such a way that accounts for the experimental observations of surface structure in postOER characterization. ${ }^{22 \mid 23}$ In Figure 3, we present this in contrast to the $\mathrm{LaCoO}_{3}$ system which experiences no such corrosion, even after extensive cycling. This materials's Pourbaix diagram suggests it has a large window of thermodynamic stability that is consistent with its experimentally observed stability.

To place this work in the appropriate context, we note that Pourbaix analysis may not tell the whole story of a given material's corrosion profile. Kinetics also play a significant role in corrosion, notably in the role of the concentrations of various salts on the conductivity of the electrolyte and therefore electrochemical rates. Additionally, the stability of a given passivation layer will frequently depend on whether its inherent strain relative to the bulk material on which it forms is energetically tolerable. If not, as predicted by the Pilling-Bedworth ratio, passivation layers will frequently flake or crack, which represents a corrosion-based mode of material failure. ${ }^{24 \mid 25}$ Furthermore, surface stabilities differ from bulk stabilities, so the profile of nearest-surface region, which may be particularly relevant to the catalytic properties of a material, may exhibit subtle differences from the profile of the 
bulk. These differences notably manifest in the role of Pourbaix-dependent surface coverage, which may influence reaction rates, particularly in alkaline OER. ${ }^{26}$ 28 Finally, we note that the quality of a given pourbaix diagram will depend on the quality of the thermodynamic data which is used to generate it. In the cases presented here, all of the input data is from DFT-computed formation energies, which have well-known and systematically correctable errors. .29 This dependence is complicated by the fact that the formation energies of ions in the Materials Project scheme are computed relative to solids in order to allow for error cancellation between ionic and solid formation energies. $\stackrel{4}{4}$ However, pymatgen's software infrastructure is agnostic to the source of a given solid formation energy, and experimental formation energies may be used alone or in concert with the computational data provided by the Materials Project API. $\stackrel{3}{ }$

In conclusion, we envision that this software functionality will have more general applications in the evaluation of corrosion resistance of complex alloys and of the stability of catalysts in high-throughput studies of water splitting and fuel cell reactions. As such, we have disseminated the implementation in the pymatgen.analysis.pourbaix_diagram module of the pymatgen open-source software, enabling its use on the Materials Project website. Ultimately, it is our hope that efficient Pourbaix analysis of these complex compounds will enable new insights to be derived on materials which were previously intractable to analyze.

Table 1: Performance Metrics for Fast Algorithm

\begin{tabular}{|c|c|c|c|c|}
\hline \multicolumn{5}{|c|}{ 2-element } \\
\hline System & Number of Fast Combos & Fast Pourbaix Time (s) & Number of Original Iterations & Original Pourbaix Time (s) \\
\hline $\mathrm{Bi}-\mathrm{V}$ & 346 & 1.1 & 49974 & 34.2 \\
\hline Sr-Ir & 203 & 1.0 & 4995 & 4.0 \\
\hline $\mathrm{Cr}-\mathrm{Fe}$ & 317 & 1.4 & 29463 & 21.0 \\
\hline Mo-S & 247 & 1.2 & 18780 & 13.6 \\
\hline \multicolumn{5}{|c|}{ 3-element } \\
\hline System & Number of Fast Combos & Fast Pourbaix Time (s) & Number of Original Iterations & Original Pourbaix Time (s) \\
\hline Fe-C-N & 748 & 1.8 & 46191274 & 29354.2 \\
\hline Zr-Ni-As & 1188 & 1.8 & 1078542 & 838.9 \\
\hline Ti-Al-Zn & 956 & 1.6 & 5979245 & 5520.0 \\
\hline Ni-C-N & 700 & 1.7 & 15879703 & 13659.0 \\
\hline \multicolumn{5}{|c|}{ 4-element } \\
\hline System & Number of Fast Combos & Fast Pourbaix Time (s) & Number of Original Iterations & Projected Original Pourbaix Time (s) \\
\hline Ba-Sr-Fe-Co & 4409 & 4.4 & 4083216060 & 3083969 \\
\hline \multicolumn{5}{|c|}{ 5-element } \\
\hline System & Number of Fast Combos & Fast Pourbaix Time (s) & Number of Original Iterations & Projected Original Pourbaix Time (s) \\
\hline Al-Mn-Fe-Cu-Mg & 26310 & 29.8 & $7.04625 \mathrm{E}+12$ & 5321889378 \\
\hline
\end{tabular}




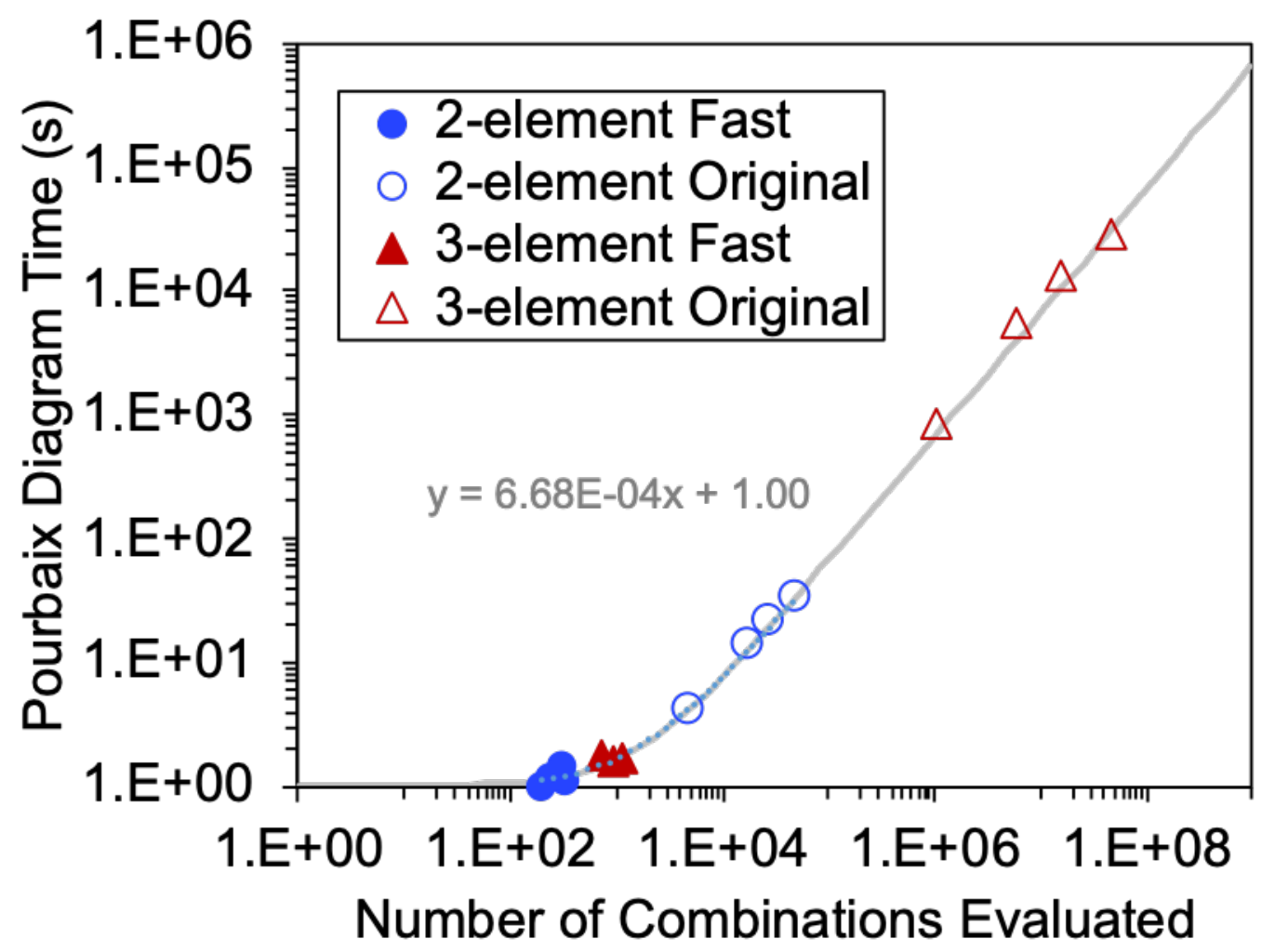

Figure 1: Relative scaling of current Pourbaix implementation, in which unfilled points represent performance and iteration count of prior pymatgen implementation of pourbaix diagram construction. Filled shapes represent performance of new implementation. Timing benchmarks for 4 and 5 element pourbaix diagrams are extrapolated based on scaling of 2 and 3-element performance with iteration count 

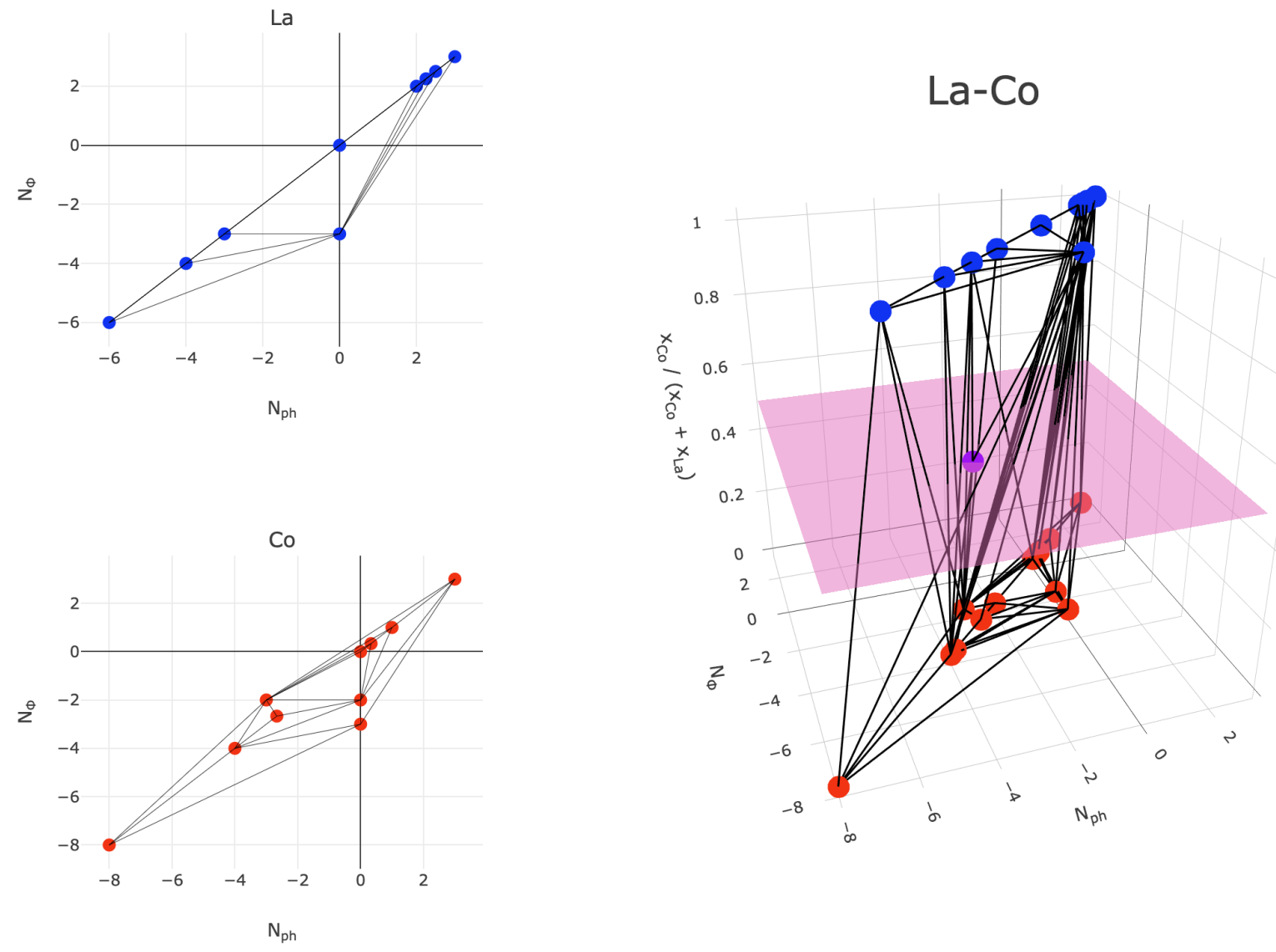

Figure 2: Convex hull projections for La, Co, and La-Co chemical systems in $N_{p H}-N_{\Phi}$ and $N_{p H}-N_{\Phi}-x_{C o}$ space. The highlighted plane in the figure corresponding to the La-Co system represents the composition constraint at a fixed non-OH composition, e. g. La:Co $=1: 1$ or $x_{C o} /\left(x_{L a}+x_{C o}\right)=0.5$. Stable combinations of entries subject to this composition constraint may only be found in the simplices of the multi-dimensional convex hull which intersect this hyperplane. Note that, in the 2-element case, mixed composition entries, for example the $\mathrm{LaCoO}_{3}$ shown in purple, appear in the interior of the simplicial complex. 

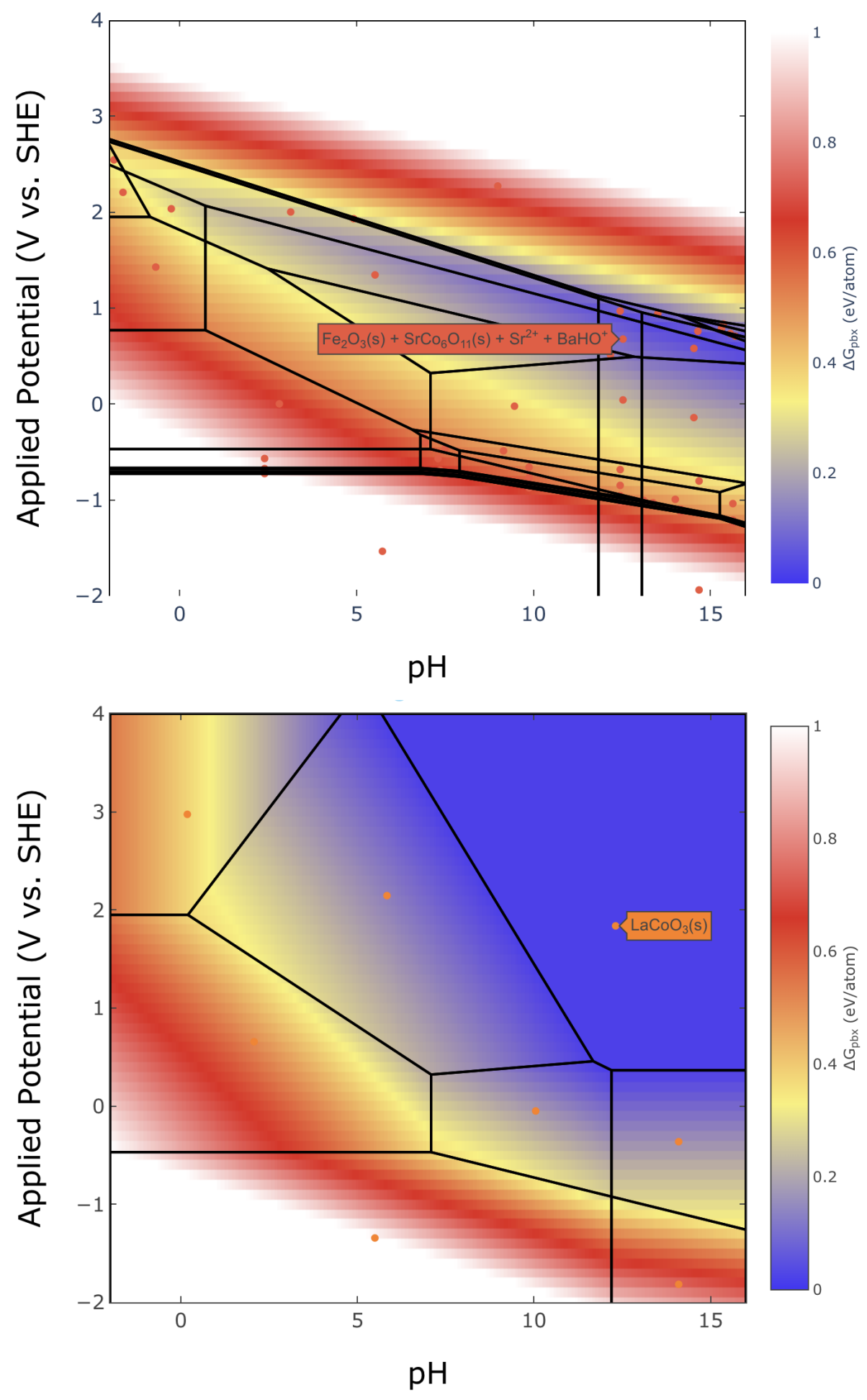

Figure 3: Pourbaix stability diagrams for (top) cubic perovskite $\mathrm{BaSrCo}_{7} \mathrm{Fe}_{7} \mathrm{O}_{24}$ (mp1075935), a model system for BSCF catalysts, and (bottom) $\mathrm{LaCoO}_{3}$. Decomposition energies in the alkaline OER region are within the metastability window $(\sim 0.1 \mathrm{eV} /$ atom $)$, and the stable phases include ion-phase $\mathrm{Sr}^{2+}$ and $\mathrm{BaHO}^{+}$, indicating a modest driving force for cation leaching, whereas $\mathrm{LaCoO}_{3}$ retains its surface structure after alkaline OER catalysis. $\frac{22123}{2}$ 


\section{References}

(1) Pourbaix, M. Corrosion Science 1974, 14, 25-82.

(2) Jain, A.; Ong, S. P.; Hautier, G.; Chen, W.; Richards, W. D.; Dacek, S.; Cholia, S.;

Gunter, D.; Skinner, D.; Ceder, G.; Persson, K. A. Commentary: The materials project:

A materials genome approach to accelerating materials innovation. 2013.

(3) Ong, S. P.; Cholia, S.; Jain, A.; Brafman, M.; Gunter, D.; Ceder, G.; Persson, K. A. Computational Materials Science 2015, 97, 209-215.

(4) Persson, K. A.; Waldwick, B.; Lazic, P.; Ceder, G. Physical Review B - Condensed Matter and Materials Physics 2012, 85.

(5) Singh, A. K.; Zhou, L.; Shinde, A.; Suram, S. K.; Montoya, J. H.; Winston, D.; Gregoire, J. M.; Persson, K. A. Chemistry of Materials 2017, 29, 10159-10167.

(6) Rossmeisl, J.; Greeley, J.; Karlberg, G. S. Fuel Cell Catalysis: A Surface Science Approach; 2008; pp 57-92.

(7) Zhou, L.; Shinde, A.; Montoya, J. H.; Singh, A.; Gul, S.; Yano, J.; Ye, Y.; Crumlin, E. J.; Richter, M. H.; Cooper, J. K.; Stein, H. S.; Haber, J. A.; Persson, K. A.; Gregoire, J. M. ACS Catalysis 2018, 8, 10938-10948.

(8) Marjolin, A.; Keith, J. A. ACS Catalysis 2015, 5, 1123-1130.

(9) Han, B.; Risch, M.; Belden, S.; Lee, S.; Bayer, D.; Mutoro, E.; Shao-Horn, Y. Journal of the Electrochemical Society 2018, 165, F813-F820.

(10) Singh, A. K.; Montoya, J. H.; Gregoire, J. M.; Persson, K. A. Nature Communications 2019, 10 .

(11) Yan, Q.; Li, G.; Newhouse, P. F.; Yu, J.; Persson, K. A.; Gregoire, J. M.; Neaton, J. B. Advanced Energy Materials 2015, 5 . 
(12) Sun, W.; Kitchaev, D. A.; Kramer, D.; Ceder, G. Nature Communications 2019, 10.

(13) Wills, L. A.; Qu, X.; Chang, I. Y.; Mustard, T. J.; Keszler, D. A.; Persson, K. A.; Cheong, P. H. Y. Nature Communications 2017, 8.

(14) Ding, R.; Shang, J. X.; Wang, F. H.; Chen, Y. Computational Materials Science 2018, 143, 431-438.

(15) Huang, L.-F.; Scully, J. R.; Rondinelli, J. M. Annual Review of Materials Research 2019, 49, 53-77.

(16) Hinnemann, B.; Moses, P. G.; Bonde, J.; Jørgensen, K. P.; Nielsen, J. H.; Horch, S.; Chorkendorff, I.; Nørskov, J. K. Journal of the American Chemical Society 2005, 127, $5308-5309$.

(17) Torelli, D. A.; Francis, S. A.; Crompton, J. C.; Javier, A.; Thompson, J. R.; Brunschwig, B. S.; Soriaga, M. P.; Lewis, N. S. ACS Catalysis 2016, 6, 2100-2104.

(18) Ong, S. P.; Richards, W. D.; Jain, A.; Hautier, G.; Kocher, M.; Cholia, S.; Gunter, D.; Chevrier, V. L.; Persson, K. A.; Ceder, G. Computational Materials Science 2013, 68, $314-319$.

(19) Thompson, W. T.; Kaye, M. H.; Bale, C. W.; Pelton, A. D. Uhlig's Corrosion Handbook: Third Edition; 2011; pp 103-109.

(20) Suntivich, J.; May, K. J.; Gasteiger, H. A.; Goodenough, J. B.; Shao-Horn, Y. Science 2011, 334, 1383-1385.

(21) Hjorth Larsen, A. et al. The atomic simulation environment - A Python library for working with atoms. 2017.

(22) Risch, M.; Grimaud, A.; May, K. J.; Stoerzinger, K. A.; Chen, T. J.; Mansour, A. N.; Shao-Horn, Y. Journal of Physical Chemistry C 2013, 117, 8628-8635. 
(23) May, K. J.; Carlton, C. E.; Stoerzinger, K. A.; Risch, M.; Suntivich, J.; Lee, Y. L.; Grimaud, A.; Shao-Horn, Y. Journal of Physical Chemistry Letters 2012, 3, 32643270.

(24) Fromhold, A. T. Theory of Metal Oxidation 1976, 1-16.

(25) Zhou, X.; Thompson, G. E.; Skeldon, P.; Wood, G. C.; Shimizu, K.; Habazaki, H. Corrosion Science 1999, 41, 1599-1613.

(26) Lee, Y. L.; Gadre, M. J.; Shao-Horn, Y.; Morgan, D. Physical Chemistry Chemical Physics 2015, 17, 21643-21663.

(27) Hansen, H. A.; Rossmeisl, J.; Nørskov, J. K. Physical Chemistry Chemical Physics 2008, 10, 3722-3730.

(28) Ulissi, Z. W.; Singh, A. R.; Tsai, C.; Nørskov, J. K. Journal of Physical Chemistry Letters 2016, 7, 3931-3935.

(29) Jain, A.; Hautier, G.; Ong, S. P.; Moore, C. J.; Fischer, C. C.; Persson, K. A.; Ceder, G. Physical Review B - Condensed Matter and Materials Physics 2011, 84. 